Gian Luca Salvagno, Gianluca Gianfilippi, Laura Pighi, Simone De Nitto, Brandon M. Henry y Giuseppe Lippi*

\title{
Evaluación de la prueba Fluorecare de anticuerpos contra la proteína Spike del SARS-CoV-2 en la práctica real
}

https://doi.org/10.1515/almed-2021-0050

Recibido 28-04-2021; aceptado 03-05-2021;

publicado en línea 14-06-2021

\section{Resumen}

Objetivos: A la luz de la elevada eficiencia diagnóstica de las pruebas rápidas de detección de antígenos (Ag-RDT) contra el coronavirus de tipo 2 causante del síndrome respiratorio agudo severo (SARS-CoV-2), se realizó un estudio con el fin de evaluar el rendimiento clínico de la prueba de antígenos contra la proteína Spike del SARS-CoV-2 en un escenario real.

Métodos: La población de estudio estaba formada por pacientes que se sometieron a una prueba diagnóstica ordinaria del SARS-Cov-2 en el Hospital Pederzoli de Peschiera del Garda (Verona, Italia). Se tomó una muestra de exudado nasofanríngeo en el momento del ingreso, que se sometió a un análisis molecular (Altona Diagnostics RealStar ${ }^{\circledR}$ SARSCoV-2 RT-PCR Kit) y de antígenos (Prueba Fluorecare de Antígenos contra la proteína Spike del SARS-CoV-2).

Resultados: La población de estudio estaba compuesta

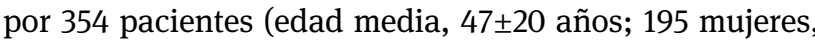
$55,1 \%)$, de los cuales $223(65,8 \%)$ obtuvieron un resultado positivo en el análisis molecular. Se observó una correlación significativa entre la prueba Fluorecare y Altona (para

\footnotetext{
*Autor para correspondencia: Prof. Giuseppe Lippi, Sección de Bioquímica Clínica, University Hospital of Verona, Universidad de Verona, Piazzale L.A. Scuro, 10, 37134, Verona, Italia, Phone: 0039045 8122970, Fax: 0039045 8124308, E-mail: giuseppe.lippi@univr.it Gian Luca Salvagno, Sección de Bioquímica Clínica, Universidad de Verona, Verona, Italia; Servicio de medicina de laboratorio, Hospital Pederzoli, Peschiera del Garda, Italia

Gianluca Gianfilippi, Dirección médica, Hospital Pederzoli, Peschiera del Garda, Italia Laura Pighi and Simone De Nitto, Sección de Bioquímica Clínica, Universidad de Verona, Verona, Italia

Brandon M. Henry, The Heart Institute, Cincinnati Children's Hospital Medical Center, Cincinnati, OH, EE.UU
}

los genes $S$ y $E$ : $\mathrm{r}=-0,75 ; \mathrm{p}<0,001)$. El área bajo la curva acumulada en todas las muestras nasofaríngeas fue de 0,68 . A un índice $S / C O \geq 1,0$, indicado por el fabricante, la sensibilidad, especifidad y valor predictivo negativo $\mathrm{y}$ positivo fueron del $27,5 \%, 99,2 \%$, 41,5\% y 98,5\%, respectivamente. La sensibilidad se mostró inversamente proporcional a los valores de Ct, llegando al 66,7\% en las muestras con valores medios de $\mathrm{Ct}<30$, al 90,5\% en aquellas con valores medios de $\mathrm{Ct}<25$, y hasta al $100 \%$ en las muestras con valores medios de $\mathrm{Ct}<20$.

Conclusiones: Dada la modesta sensibilidad y moderado valor predictivo negativo de la prueba de Fluorecare, no se recomienda sustituir con esta prueba el análisis molecular para el diagnóstico de infección por SARS-CoV-2, aunque su adecuada sensibilidad confirma su fiabilidad para el cribado de pacientes con mayor potencial infeccioso.

Palabras clave: COVID-19; SARS-CoV-2; medicina de laboratorio; diagnóstico; inmunoensayo.

\section{Introducción}

Ante la enorme presión soportada por los laboratorios diagnósticos a consecuencia de la actual pandemia de la enfermedad por coronavirus 2019 (COVID-19), las pruebas rápidas de antígenos se postulan como una alternativa para la identificación masiva, rápida y eficiente de pacientes con síndrome respiratorio agudo severo provocado por infección por coronavirus tipo 2 (SARS-CoV-2) [1]. No obstante, tal como recientemente han respaldado la Organización Mundial de la Salud (OMS) [2] y el grupo de trabajo sobre COVID-19 de la Federación Internacional de Química Clínica y Medicina de Laboratorio (IFCC) [3], se debe evaluar concienzudamente el rendimiento clínico de cada prueba de detección rápida de antígenos contra SARS-CoV-2 (Ag-RDT), previamente a su introducción en cualquier nivel de la práctica clínica. El objeto de este estudio es evaluar el rendimiento clínico de la prueba de antígenos contra la proteína Spike del SARS-CoV-2 (en adelante, Fluorecare) en un escenario real. 


\section{Materiales y métodos}

\section{Población de estudio}

Nuestra población de estudio estaba compuesta por pacientes que se sometieron a las pruebas diagnósticas de SARS-Cov-2 en el Servicio de Medicina de Laboratorio del Hospital Pederzoli (Peschiera del Garda, Verona, Italia) entre el 2 y el 19 de abril de 2021, por encontrarse sintomáticos o haber estado en contracto estrecho con otros pacientes con COVID-19. Se tomó inmediatamente un exudado nasofaríngeo (Virus swab UTM ${ }^{\mathrm{TM}}$, Copan, Brescia, Italia) en el momento del ingreso, que se sometió a un análisis molecular y de antígenos.

\section{Análisis molecular}

La prueba de amplificación de ácidos nucleicos de SARS-CoV-2 (NAAT) se realizó con la prueba Altona Diagnostics RealStar ${ }^{\circledR}$ SARSCoV-2 RT-PCR (Altona Diagnostics GmbH, Hamburg, Alemania). Esta reacción en cadena de la polimerasa con transcriptasa inversa (rRT-PCR) consiste en dos amplificaciones y detecciones dirigidas cada una a las secuencias genéticas $E$ y $S$ del SARS-CoV-2. Para detectar una posible inhibición en la rRT-PCR, incluye como control interno una sonda y un cebador. La prueba se realizó con un sistema de detección Bio-Rad CFX96 ${ }^{\mathrm{TM}}$ Deep Well Dx Real-Time PCR Detection System (Bio-Rad Laboratories, Hercules, CA, EE.UU). El resultado era positivo si se obtenían valores del umbral de ciclo (Ct) inferiores a 45 para los genes $S$ y $E$ del SARS-CoV-2.

\section{Prueba de antígenos}

La prueba de antígenos se realizó con la prueba inmunocromatográfica Fluorecare de anticuerpos contra la proteína Spike del SARS-CoV-2 (Microprofit Biotech, Shenzhen, China). Explicado brevemente, se mezcla una muestra nasofaríngea con una solución de anticuerpos contra la proteína Spike del SARS-CoV-2 marcados con fluorocromos. Los inmunocomplejos resultantes migran a través de la membrana nitrocelulosa al área de detección, donde generan una línea roja (en las muestras positivas para la proteína Spike del SARS-CoV-2). Los anticuerpos contra la proteína Spike del SARS-CoV-2 marcados con fluorocromos también migran hacia la ventana de control de calidad, donde se genera otra línea para verificar que la prueba funciona correctamente. La realización de la prueba completa lleva entre 15 y 30 minutos. Para la lectura cuantitativa de fluorescencia se pueden emplear instrumentos en el mismo punto de atención (Fluorecare MF-T1000; Microprofit Biotech, Shenzhen, China), y se refleja como una unidad de medida arbitraria (esto es, índice $\mathrm{S} / \mathrm{CO}$ ). La prueba es positiva si el índice $\mathrm{S} / \mathrm{CO}$ es $\geq 1,0$. Según el fabricante, los porcentajes de concordancia positivo y negativo usando la RT-PCR como referencia son del $92,2 \%$ y el $100 \%$, respectivamente.

\section{Análisis estadístico}

En nuestro estudio, el rendimiento diagnóstico de Fluorecare frente al NAAT, empleado como referencia, se evaluó mediante la correlación de Spearman, construyendo curvas ROC y calculando su sensibilidad, especifidad, valor predictivo negativo (VPN) y valor predictivo positivo (VPP). El análisis estadístico se realizó con el programa Analyse-it (Analyse-it Software Ltd, Leeds, UK). La investigación se realizó como parte de las actividades del laboratorio clínico, empleando muestras ya existentes tomadas para el diagnóstico del SARS-CoV-2 en el centro, por lo que no fue necesario obtener el consentimiento informado del paciente ni aprobación por parte del Comité Ético. Los resultados de todas las pruebas fueron anonimizados previamente a su análisis estadístico. El estudio se realizó de conformidad con los principios de la Declaración de Helsinki, respetando la legislación nacional pertinente.

\section{Resultados}

La población final de estudio estaba compuesta por 354 pacientes (edad media, $47 \pm 20$ años; 195 mujeres, $55,1 \%), 223$ de los cuales $(65,8 \%)$ fueron positivos en NAAT (i.e., valores de $\mathrm{Ct}<45$ para los genes $S$ y $E$ del SARS-CoV-2). Los valores de $\mathrm{Ct}$ de las muestras positivas fueron $29,8 \pm 7,1$ y 30,3 \pm 7,0 para los genes $S$ y E del SARS-CoV-2, respectivamente. Observamos una correlación notablemente significativa entre los valores de Fluorecare y los valores de Ct evaluables de los genes $S$ $(\mathrm{r}=-0,75$; IC95\%, entre $-0,80$ y $-0,69 ; \mathrm{p}<0,001)$ y $E$ $(\mathrm{r}=-0,75$; IC95\%, entre $-0,80 \mathrm{y}-0,68 ; \mathrm{p}<0,001)$.

El rendimiento diagnóstico global de Fluorecare, así como su rendimiento estratificado por valores de Ct de Altona se muestran en la Tabla 1. El área bajo la curva (AUC) acumulada en todas las muestras nasofaringeas fue de 0,68 . Aplicando el punto de corte $\geq 1,0$ para al índice $\mathrm{S} / \mathrm{CO}$ indicado por el fabricante, la sensibilidad, especifidad, y valores VPN y VPP fueron del 27,5\%, 99,2\%, 41,5\% y $98,5 \%$, respectivamente. El mejor punto de corte para $\mathrm{S} / \mathrm{CO}$, calculado a partir de la curva ROC, fue 0,18 , asociado con una sensibilidad del 42,9\% (IC95\%, 36,5-49,5\%), una especifidad del 92,6\% (IC95\%, 86,4-96,5\%), un VPP del 45,7\% (IC95\%, 42,7-48,8\%), un VPN del 91,7\% (IC 95\%, 85,4-95,5\%). La sensibilidad aumentaba notablemente con valores bajos de $\mathrm{Ct}$, llegando al $66,7 \%$ en las muestras con valores medios de $\mathrm{Ct}<30,90,5 \%$ en aquellas con valores de Ct medios $<25$, y hasta el $100 \%$ en aquellas con valores medios de $\mathrm{Ct}<20$.

En la Figura 1 se muestra la distribución de los valores obtenidos con Fluorecare con respecto a los umbrales de Altona para positividad de la muestra (esto es, valores de $\mathrm{Ct}<45$ de los genes $S$ y $E$ del SARS-CoV-2) o mayor riesgo de contagiosidad (valores de $\mathrm{Ct}<26,3$ de los genes $S$ y $E$ del SARS-CoV-2). El valor medio obtenido con Fluorecare SARS-CoV-2 fue significativamente mayor en las muestras con valores de $\mathrm{Ct}<45$ ( $\mathrm{n}=233$; 2,1 $\pm 3,6 \mathrm{~S} / \mathrm{CO})$, frente las muestras con valores de $C t$ superiores $(n=121$; 
Tabla 1: Rendimiento clínico de la prueba Fluorecare de anticuerpos contra la proteína Spike del SARS-CoV-2 estratificado por valores del umbral de ciclo $(\mathrm{Ct})$.

\begin{tabular}{|c|c|c|c|c|c|c|}
\hline Valores Ct & n & AUC & SEN & ESP & VPN & VPP \\
\hline Todas las muestras & 354 & $\begin{array}{l}0,68 \\
(\text { IC95\%, 0,62-0,73) }\end{array}$ & $\begin{array}{l}27,5 \% \\
\text { (IC95\%, 21,8-33,7\%) }\end{array}$ & $\begin{array}{l}99,2 \% \\
\text { (IC95\%, 95,5-100\%) }\end{array}$ & $\begin{array}{l}41,5 \% \\
\text { (IC95\%, 39,6-43,5\%) }\end{array}$ & $\begin{array}{l}98,5 \% \\
\text { (IC95\%, 90,0-99,8\%) }\end{array}$ \\
\hline$<30$ & 96 & - & $\begin{array}{l}66,7 \% \\
\text { (IC95\%, 56,3-76,0\%) }\end{array}$ & - & - & - \\
\hline$<25$ & 63 & - & $\begin{array}{l}90,5 \% \\
\text { (IC95\%, 80,4-96,4\%) }\end{array}$ & - & - & - \\
\hline$<20$ & 36 & - & $\begin{array}{l}100 \% \\
\text { (IC95\%, 90,3-100\%) }\end{array}$ & - & - & - \\
\hline
\end{tabular}

AUC, área bajo la curva; SEN, sensibilidad; ESP, especifidad; VPN, valor predictivo negativo; VPP, valor predictivo positivo.

$0,1 \pm 0,1 \mathrm{~S} / \mathrm{CO} ; \mathrm{p}<0,001)$, así como en aquellas con valores de $\mathrm{Ct}<26,3$ ( $\mathrm{n}=68 ; 6,7 \pm 4,0 \mathrm{~S} / \mathrm{CO}$ ), frente a las muestras con valores de $\mathrm{Ct}$ superiores a este rango $(n=286 ; 0,2 \pm 0,2 \mathrm{~S} / \mathrm{CO}$; $\mathrm{p}<0,001)$.

\section{Discusión}

El antígeno de SARS-CoV-2, principalmente representado por Ag-RDT, se postula como una posible alternativa que permitiría aliviar la enorme presión que sufren los laboratorios clínicos con motivo de las numerosas pruebas para el diagnóstico de la COVID-19 [1, 4, 5]. Un análisis reciente del Grupo Cochrane de Precisión de Pruebas Diagnósticas para la COVID-19 subraya las aparentes ventajas y posibles inconvenientes de estas técnicas [6]. Con respecto a sus

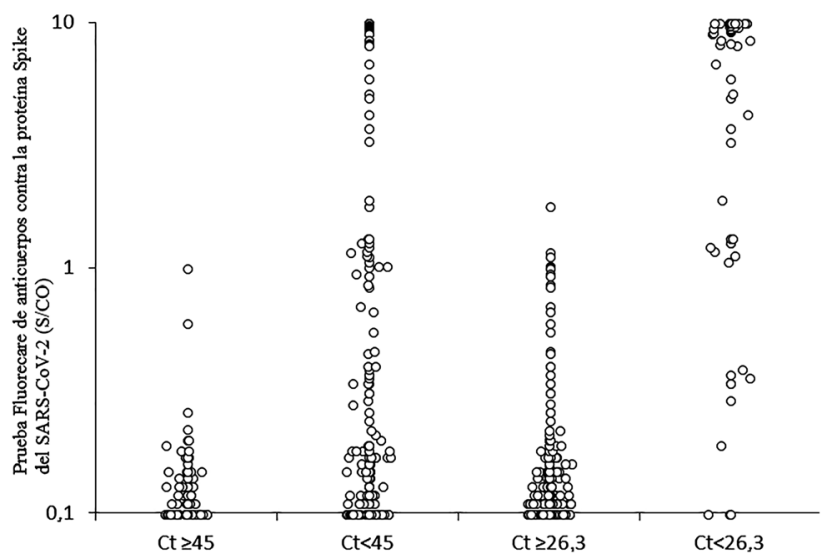

Figura 1: Distribución de los valores obtenidos con Fluorecare aplicando los umbrales de Altona para positividad de la muestra (esto es, valores de $\mathrm{Ct}<45$ de los genes $S$ y $E$ del SARS-CoV-2) 0 mayor riesgo de infectividad (esto es, valores de $\mathrm{Ct}<26,3$ de los genes $S$ y $E$ del SARS-CoV-2).

$\mathrm{Ct}$, umbral de ciclo; SARS-CoV-2, coronavirus del síndrome respiratorio agudo grave; $\mathrm{S} / \mathrm{CO}$, índice $\mathrm{S} / \mathrm{CO}$. ventajas, estas pruebas son sencillas, las puede realizar cualquier persona que haya recibido una breve instrucción o incluso los propios pacientes; no precisan el uso de analizadores de los laboratorios centrales y ofrecen resultados manejables de una forma rápida. Sin embargo, el rendimiento clínico de los antígenos Ag-RDT sigue siendo considerablemente inferior al del análisis molecular, y su sensibilidad diagnóstica acumulada no logra superar el $60-75 \%$ [6]. Se ha demostrado que posee un rendimiento especialmente discreto en muestras con una baja carga viral (valores de $\mathrm{Ct} \geq 25$ ), con una sensibilidad diagnóstica de entre el 30 y el 50\%, que aumenta a entre el 91 y el $97 \%$ en las muestras con mayor carga viral (valores de $\mathrm{Ct}<25$ ). Sin embargo, el mismo Grupo Cochrane ha hecho notar la amplia heterogeneidad en el rendimiento diagnóstico de las diferentes pruebas, con sensibilidades que oscilan entre extremadamente bajas (e.g. 12\%) y excepcionalmente elevadas (e.g. 90\% o incluso superiores). Este escenario incierto ha llevado a la OMS y al Grupo de Trabajo sobre COVID-19 del IFCC a recomendar que cada Ag-RDT se someta a un proceso local de validación, previamente a su introducción en la práctica clínica [2, 3].

Los resultados de este estudio tienen dos implicaciones principales. En primer lugar, la relativamente modesta sensibilidad global y VPN de la prueba Fluorecare de la proteína Spike del SARS-CoV-2 muestra que esta prueba no puede sustituir ni es una alternativa al NAAT para el diagnóstico de la infección por SARS-CoV-2. No obstante, su satisfactoria sensibilidad en las muestras con elevada carga viral sí la hace una prueba adecuada para el cribado de pacientes con un mayor potencial infeccioso. Un estudio reciente publicado por Gniazdowski y col [7]. revela que la probabilidad de obtener un cultivo positivo para SARS-CoV-2 fue $<3 \%$ en las muestras nasofaríngeas con valores de Ct de Altona <26,2. Aplicando un umbral de $\mathrm{Ct}$ similar, Fluorecare mostró una sensibilidad diagnóstica del $90 \%$ en nuestra cohorte de pacientes no seleccionados 
sometidos a una prueba diagnóstica ordinaria de COVID-19, lo que habría permitido identificar a la gran mayoría de los pacientes con una elevada carga viral en el tracto respiratorio superior, que son los que representan un mayor riesgo de expandir el virus en la comunidad (los llamados "supercontagiadores") [8].

Otro aspecto importante a tener en cuenta es que la carga viral nasofaríngea no solo influye en la probabilidad de contagio del SARS-CoV-2 entre individuos, sino que también está relacionada con el riesgo de progresión de COVID-19 a enfermedad grave o crítica y/o mortalidad, tal como ya han demostrado estudios anteriores [9-11]. De este modo, la identificación rápida y eficaz y/o el seguimiento de los pacientes con COVID-19 con una elevada carga nasofaríngea de SARS-CoV-2 durante el triaje (incluso fuera de los centros sanitarios), así como durante la hospitalización o en el momento del ingreso en la UCI, permitiría establecer un tratamiento más agresivo en el momento oportuno, aumentando así la probabilidad de obtener un buen resultado clínico de esta enfermedad potencialmente mortal.

En conclusión, los hallazgos de este estudio indican que es preferible reservar el uso de esta Ag-RDT para identificar a los pacientes con una mayor carga viral.

Agradecimientos: Los autores agradecen al personal del Servicio de Medicina de Laboratorio del Hospital Pederzoli (Peschiera del Garda, Italia) por su asistencia técnica.

Financiación de la investigación: No declarada.

Contribución de los autores: Todos los autores aceptan su responsabilidad en relación al contenido del manuscrito y aprueban su presentación.

Conflicto de intereses: Los autores declaran no tener ningún conflicto de intereses.

Consentimiento informado: La investigación se realizó como parte de las actividades del laboratorio clínico, empleando muestras ya existentes tomadas para el diagnóstico del SARS-CoV-2 en el centro, por lo que no fue necesario obtener el consentimiento informado del paciente ni aprobación por parte del Comité Ético.

Aprobación ética: La investigación se realizó como parte de las actividades del laboratorio clínico, empleando muestras ya existentes tomadas para el diagnóstico del SARS-CoV-2 en el centro, por lo que no fue necesario obtener el consentimiento informado del paciente ni aprobación por parte del Comité Ético.

\section{Referencias}

1. Mattiuzzi C, Henry BM, Lippi G. Making sense of rapid antigen testing in severe acute respiratory syndrome coronavirus 2 (SARS-CoV-2) diagnostics. Diagnosis 2020;8:27-31.

2. World Health Organization. Antigen-detection in the diagnosis of SARS-CoV-2 infection using rapid immunoassays; 2020.

3. Bohn MK, Lippi G, Horvath AR, Erasmus R, Grimmler M, Gramegna $M$, et al. IFCC interim guidelines on rapid point-of-care antigen testing for SARS-CoV-2 detection in asymptomatic and symptomatic individuals. Clin Chem Lab Med 2021. https://doi. org/10.1515/cclm-2021.0455.

4. Lippi G. Brief update on coronavirus disease 2019 (COVID-19) diagnostics. Adv Lab Med 2020;1:20200099. https://doi.org/10. 1515/almed-2020-0099.

5. Lippi G, Plebani M. Laboratory medicine resilience during coronavirus disease 2019 (COVID-19) pandemic. Adv Lab Med 2020: 20200035. https://doi.org/10.5935/1676-2444.20200035.

6. Dinnes J, Deeks JJ, Berhane S, Taylor M, Adriano A, Davenport C, et al. Rapid, point-of-care antigen and molecular-based tests for diagnosis of SARS-CoV-2 infection. Cochrane Database Syst Rev 2021;3:CD013705. https://doi.org/10.1002/14651858. CD013705.

7. Gniazdowski V, Morris CP, Wohl S, Mehoke T, Ramakrishnan S, Thielen P, et al. Repeat COVID-19 molecular testing: correlation of SARS-CoV-2 culture with molecular assays and cycle thresholds. Clin Infect Dis 2020 Oct 27:ciaa1616. https://doi.org/10.1093/ cid/ciaa1616 [Epub ahead of print].

8. Goyal A, Reeves DB, Cardozo-Ojeda EF, Schiffer JT, Mayer BT. Viral load and contact heterogeneity predict SARS-CoV-2 transmission and super-spreading events. Elife 2021;10:e63537.

9. Westblade LF, Brar G, Pinheiro LC, Paidoussis D, Rajan M, Martin $P$, et al. SARS-CoV-2 viral load predicts mortality in patients with and without cancer who are hospitalized with COVID-19. Canc Cell 2020;38:661-71.

10. Pujadas E, Chaudhry F, McBride R, Richter F, Zhao S, Wajnberg A, et al. SARS-CoV-2 viral load predicts COVID-19 mortality. Lancet Respir Med 2020;8:e70.

11. Alteri C, Cento V, Vecchi M, Colagrossi L, Fanti D, Vismara C, et al., SCOVA Study Group. Nasopharyngeal SARS-CoV-2 load at hospital admission as predictor of mortality. Clin Infect Dis 2020 Jul 16:ciaa956. https://doi.org/10.1093/cid/ciaa956 [Epub ahead of print].

Nota del artículo: El artículo original puede encontrarse aquí: https:// doi.org/10.1515/almed-2021-0041. 